\title{
An Interrupted Sampling Scattered Wave Deception Jamming Method against Three-Channel SAR GMTI
}

\author{
Xin Chang $\left(\mathbb{D},{ }^{1}\right.$ Chunxi Dong $\mathbb{D}^{1},{ }^{1}$ Gao Weichen $\mathbb{D D}^{2}$, and Yan Zhao $\mathbb{D D}^{1}$ \\ ${ }^{1}$ School of Electronic Engineering, Xidian University, Xi'an 710071, China \\ ${ }^{2}$ Beijing Sinotech Information Technology Company Limited, Beijing 100085, China \\ Correspondence should be addressed to Xin Chang; changxinydb@163.com
}

Received 19 January 2020; Revised 21 June 2020; Accepted 26 June 2020; Published 27 July 2020

Academic Editor: Haiyan Lu

Copyright (c) 2020 Xin Chang et al. This is an open access article distributed under the Creative Commons Attribution License, which permits unrestricted use, distribution, and reproduction in any medium, provided the original work is properly cited.

An important problem is how to generate false moving targets, whose relocated azimuth position is similar to that of real moving targets. To solve this problem, an interrupted sampling scattered wave deception jamming method against threechannel synthetic aperture radar ground moving target indication (SAR GMTI) is proposed. A stationary jammer uses a controllable jammer antenna to generate verisimilar moving targets by controlling velocity and initial position of jammer beam footprint. The antenna sampled moves along the different tracks. For each track, the slant history of jamming signal is changed varying with different pulse recurrence intervals (PRI), and the movement of the footprint will introduce a Doppler frequency in jamming the signal. By analyzing parameters' difference between echoes and jamming signal, the velocity and the initial position of the footprint will be calculated, and then the verisimilar false targets are generated. The effectiveness of the method is verified by simulation experiments.

\section{Introduction}

Synthetic aperture radar ground moving target indication (SAR GMTI) is able to get high resolution images of sensitive areas and information of moving targets at all-time under all-weather condition [1-4]. Displaced phase center antenna (DPCA) and along track interferometry (ATI) are a type of three-channel SAR GMTI. DPCA technique can detect slow moving targets, and ATI technique can acquire velocity of moving targets. Furthermore, ATI technique can obtain the relocated azimuth position of targets which can be used to relocate the moving target to the correct positions and distinguish real moving targets and false targets [5-8]. Because of their ease of use, they are widely used in civilian and military field. So, an important research topic is how to effectively protect information of moving targets against DPCA and ATI techniques [9-11]. In addition, research on jamming and antijamming method will be on the improved radar system in the future $[12,13]$.

The jamming methods are generally classified into barrage jamming methods and deception jamming methods. High noise-like jamming signal is retransmitted to cover the working band of SAR, and then a signal-tonoise ratio (SNR) is reduced in whole SAR images to prevent detecting targets from three-channel SAR GMTI. However, the barraged jamming method requires higher power than a deception jamming method to achieve a low signal-to-noise ratio (SNR) in SAR imaging results $[14,15]$. In addition, because the azimuth jammer filter is generated by utilizing DPCA technique, the false target will be cancelled near the jammer. So, an improved barraged jamming method, which uses two jammers, is used to cover the whole DPCA images [16]. However, the distance between the jammers is also required to control and set before the jamming area, and this reduces the flexibility of the method. According to the path of jamming signal, deception jamming includes direct-path deception jamming methods and multipath deception jamming methods. In direct-path jamming methods, the jammer generates false targets by intercepting, modulating, and retransmitting radar signal. However, because false targets are all generated by a stationary jammer, false targets near the jammer 
will be cancelled by DPCA technique and those all have the same relocated azimuth position by ATI technique [16-21]. This result is obviously different from the detecting result of moving targets, and false targets are easy to be recognized. To solve this disadvantage, deception jamming methods is proposed by utilizing multiple coherent jammers $[9,22,23]$. The phase difference of jamming signal in the different received antenna of the radar is controlled to adjust against the suppression of interferometric synthetic aperture radar (InSAR) and SAR GMTI. Every false target has the desired relocated azimuth position. However, because the phase is needed to be summed by every multiple jammers, it is difficult to control the additional phase terms in the received antenna. In addition, the distance between the jammers also need to control and set before SAR working. In other way, scattered wave jamming is a kind of multipath jamming method. The intercepted signal is scattered by scatterers of the ground to form jamming signal [10, 24-26]. In our previous work, a novel method is proposed to generate a verisimilar moving target by controlling a jammer velocity and a jammer beam footprint [27]. Although this method can effectively generate a verisimilar moving target after DPCA technique, there are some problems that we need to further address as follows. First, sometimes, it is difficult to control a moving jammer with the desired accurate speed, and the jammer speed always has a relatively large error compared with ideal speed. Second, after ATI technique, multiple false moving targets generated with this method cannot simulate the multiple moving targets with the desired relocated azimuth positions. Final, jamming effect against ATI technique is not analyzed.

An important problem is how to generate false moving targets, whose relocated azimuth positions are similar to the relocated azimuth position of real moving targets. So, an improved deception jamming method with a controllable jammer antenna is proposed to solve this problem. The improvement is that a stationary jammer with a controllable jammer antenna. The footprint moves in the calculated initial position and at a calculated velocity, and then the track is formed. The intercepted and retransmitted signal is scattered by the scattered points on the ground to form jamming signal. The jammer beam footprint intercepted sampling moves along the different tracks to generate multiple false moving targets. For each track, the slant history of jamming signal is changed varying with different pulse recurrence intervals (PRI), and the movement of the footprint will introduce a Doppler frequency in the retransmitted jamming signal. By analyzing parameter difference between the false target and the moving target, including position, Doppler rate, amplitude after DPCA technique, and the relocated azimuth position after ATI technique, the velocity and initial position of the footprint are calculated to determine the track, and then jamming signal forms a verisimilar false moving targets.

The remainder of this paper is organized as follows. Section 2 introduces the geometry of the proposed method. The difference between echo of the moving target and the jamming signal are also compared. In Section 3, parameter difference between a real moving target and a false target is analyzed. In addition, the process of selecting the position and velocity of footprint is also summarized to eliminate the difference and generate verisimilar false moving targets. Section 4 gives experiment results of the proposed method compared with a traditional deception jamming method. In Section 5, conclusion is given.

\section{Geometry of a Scattered Wave Deception Jamming Method Based on Controllable Jammer Antenna}

As shown in Figure 1, radar flies along with $X$-axis, which is the azimuth direction. Its ideal velocity is $v$ and its altitude is $H$. $Y$-axis is the ground range direction, and $Z$-axis is the altitude direction.

The three antenna of radar are denoted by the $A_{1}, A_{2}$, and $A_{3}$, and their coordinates are $\left(v t_{a}-D, 0, H\right),\left(v t_{a}, 0, H\right)$, and $\left(v t_{a}+D, 0, H\right)$, respectively. $t_{a}$ is slow time and $D$ is a distance among antenna. Radar transmits signal from antenna $A_{1}$ and antenna $A_{2} . A_{3}$ receives echo. $P$ is a moving target, and its coordinate is $\left(x_{P}+v_{x} t_{a}, y_{P}+v_{y} t_{a}, 0\right)$. $J$ is a stationary jammer, and its coordinate is $\left(x_{J}, y_{J}, h\right)$. The intercepted signal is transmitted and amplified by $J$, and then it is illuminated to the area under the jammer beam. By controlling the jammer beam, the jammer beam footprint moves at a calculated velocity with a calculated initial position. $I$ is the center of the footprint and its coordinate is $\left(x_{I}+v_{x}^{\prime} t_{a}\right.$, $\left.y_{I}+v_{y}^{\prime} t_{a}, 0\right)$. The intercepted signal scattered by the scattered points covered by $I$ to form the jamming signal. By adjusting parameters of the velocity and the initial position, jamming signal will be similar to echo, and then $F$ will be similar to $P$.

2.1. Echoes. The $P^{\prime}$ 's slant range of antenna $A_{1}$ and $A_{3}$ are, respectively, given by

$$
\begin{aligned}
R_{P_{1}}\left(t_{a}\right) & =R_{P A_{2}}\left(t_{a}\right)+R_{P A_{1}}\left(t_{a}\right) \\
R_{P_{3}}\left(t_{a}\right) & =R_{P A_{2}}\left(t_{a}\right)+R_{P A_{3}}\left(t_{a}\right) \\
R_{P A_{1}}\left(t_{a}\right) & =\sqrt{\left(x_{P}+v_{x} t_{a}-v t_{a}+D\right)^{2}+\left(y_{P}+v_{y} t_{a}\right)^{2}+H^{2}} \\
R_{P A_{2}}\left(t_{a}\right) & =\sqrt{\left(x_{P}+v_{x} t_{a}-v t_{a}\right)^{2}+\left(y_{P}+v_{y} t_{a}\right)^{2}+H^{2}} \\
R_{P A_{3}}\left(t_{a}\right) & =\sqrt{\left(x_{P}+v_{x} t_{a}-v t_{a}-D\right)^{2}+\left(y_{P}+v_{y} t_{a}\right)^{2}+H^{2}} .
\end{aligned}
$$

Equations (3)-(5) can be expanded as a Taylor series around $t_{a}$ at $D / v, 0$, and $-(D / v)$, and then they are, respectively, given by 


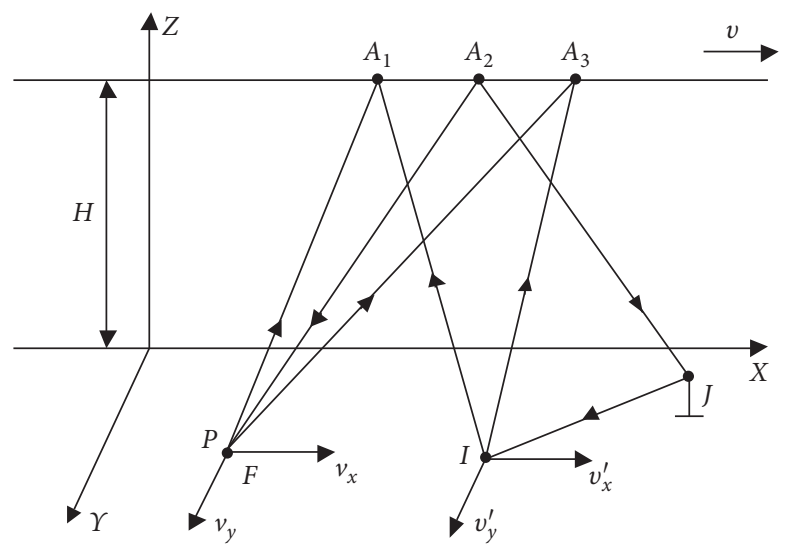

Figure 1: Geometry of the proposed method.

$$
\begin{aligned}
R_{P A_{1}}\left(t_{a}\right) & =R_{1}+\frac{\left[\left(v_{x}-v\right)\left(x_{P}+\left(v_{x} D / v\right)\right)+\left(y_{P}+\left(v_{y} D / v\right)\right) v_{y}\right]\left(t_{a}-(D / v)\right)}{R_{1}}+\frac{\left[\left(v-v_{x}\right)^{2}+v_{y}^{2}\right]}{2 R_{1}}\left(t_{a}-\frac{D}{v}\right)^{2}, \\
R_{P A_{2}}\left(t_{a}\right) & =R_{1}+\frac{\left[x_{P}\left(v_{x}-v\right)+y_{P} v_{y}\right]}{R_{1}} t_{a}+\frac{\left[\left(v-v_{x}\right)^{2}+v_{y}^{2}\right]}{2 R_{1}} t_{a}^{2}, \\
R_{P A_{3}}\left(t_{a}\right) & =R_{1}+\frac{\left[\left(v_{x}-v\right)\left(x_{P}-\left(v_{x} D / v\right)\right)+\left(y_{P}+\left(v_{y} D / v\right)\right) v_{y}\right]\left(t_{a}+(D / v)\right)}{R_{1}}+\frac{\left[\left(v-v_{x}\right)^{2}+v_{y}^{2}\right]}{2 R_{1}}\left(t_{a}+\frac{D}{v}\right)^{2}, \\
R_{1} & =\sqrt{x_{P}^{2}+y_{P}^{2}+H^{2}} .
\end{aligned}
$$

Because DPCA and ATI techniques are used to detect the slow moving target, which means $P$ is always slower than radar, $v_{x} \ll v, v_{y} \ll v$, and $D \ll R_{2}$ can be hold. Then, the slant range can be, respectively, given by

$$
\begin{aligned}
& R_{P_{1}}\left(t_{a}\right)=2 R_{P A_{2}}\left(t_{a}\right)+\frac{x_{P} D}{R_{1}}-\frac{v D}{R_{1}} t_{a}+\frac{D^{2}}{2 R_{1}}, \\
& R_{P_{3}}\left(t_{a}\right)=2 R_{P A_{2}}\left(t_{a}\right)-\frac{x_{P} D}{R_{1}}+\frac{v D}{R_{1}} t_{a}+\frac{D^{2}}{2 R_{1}} .
\end{aligned}
$$

Linear frequency modulation signal is used by the radar as follows:

$$
S_{0}\left(t_{r}, t_{a}\right)=a_{r}\left(t_{r}\right) \exp \left\{j 2 \pi\left[f_{0}\left(t_{r}+t_{a}\right)+\frac{\gamma t_{a}^{2}}{2}\right]\right\},
$$

where $t_{r}$ is fast time, $f_{0}$ is center frequency, $\gamma$ is chirp rate, $a_{r}\left(t_{r}\right)=\left\{\begin{array}{ll}1, & \left|t_{r}\right| \leq T_{r} / 2, \\ 0, & \left|t_{r}\right|>T_{r} / 2,\end{array}\right.$ is pulse envelope, and $T_{r}$ is pulse duration.
The baseband echo from $P$ of antenna $A_{1}$ and $A_{3}$ are, respectively, expressed as follows:

$$
\begin{aligned}
S_{P_{1}}\left(t_{r}, t_{a}\right)= & \sigma_{P} a_{r}\left(t_{r}-\frac{R_{P_{1}}\left(t_{a}\right)}{c}\right) a_{a z}\left(t_{a}-\frac{x_{P}^{\prime}}{v}\right) \\
& \cdot \exp \left[j \pi \gamma\left(t_{r}-\frac{R_{P_{1}}\left(t_{a}\right)}{c}\right)^{2}\right] \exp \left[-j \frac{2 \pi}{\lambda} R_{P_{1}}\left(t_{a}\right)\right], \\
S_{P_{3}}\left(t_{r}, t_{a}\right)= & \sigma_{P} a_{r}\left[t_{r}-\frac{R_{P_{3}}\left(t_{a}\right)}{c}\right] a_{a z}\left(t_{a}-\frac{x_{P}^{\prime}}{v}\right) \\
& \cdot \exp \left[j \pi \gamma\left(t_{r}-\frac{R_{P_{3}}\left(t_{a}\right)}{c}\right)^{2}\right] \exp \left[-j \frac{2 \pi}{\lambda} R_{P_{3}}\left(t_{a}\right)\right],
\end{aligned}
$$

where $\lambda$ is wavelength, $\sigma_{P}$ is radar cross section (RCS) of $P$, $a_{a z}\left(t_{a}\right)=\left\{\begin{array}{ll}1, & \left|t_{a}\right| \leq T_{a} / 2, \\ 0, & \left|t_{a}\right|>T_{a} / 2,\end{array}\right.$ is azimuth envelope, $x_{P}^{\prime}$ is azimuth position of $P$, and $T_{a}$ is target exposure time. 
2.2. Jamming Signal. To generate moving targets at the same time, the jammer beam interrupted sampling scans and illuminates the ground along the different tracks, as shown in Figure 2, which tracks are determined by the footprint parameters of the velocity and the initial position, and then the verisimilar false moving targets will be generated. For example, the jammer beam footprint interrupted sampling scans and illuminates along two tracks.

As shown in Figure 3, PRI is pulse recurrence interval, $\tau=$ PRI is the slice width and the duration of illumination for each track, and $T=N \cdot$ PRI is the time interval between adjacent intercepted slice, where $N$ presents the number of tracks. During $T$, the jammer beam can illuminate different tracks to form multiple targets.

In other words, the jamming signal is likely performed by interrupted sampling modulation, as shown in Figure 3 [28]. It can be given by

$$
P\left(t_{a}\right)=\operatorname{rect}\left(\frac{t_{a}}{\tau}\right) * \sum_{l=-\infty}^{+\infty} \delta\left(t_{a}-l T\right)
$$

where $\operatorname{rect}(\cdot)$ is the rectangular window function.

Slant ranges of jamming signal received by antennae $A_{1}$ and $A_{3}$ are, respectively, expressed as follows:

$$
\begin{aligned}
& R_{F_{1}}\left(t_{a}\right)=R_{A_{2} J}\left(t_{a}\right)+R_{J I}\left(t_{a}\right)+R_{I A_{1}}\left(t_{a}\right), \\
& R_{F_{3}}\left(t_{a}\right)=R_{A_{2} J}\left(t_{a}\right)+R_{J I}\left(t_{a}\right)+R_{I A_{3}}\left(t_{a}\right),
\end{aligned}
$$

$$
\begin{aligned}
R_{A_{2} J}\left(t_{a}\right) & =R_{2}-\frac{x_{J} v}{R_{2}} t_{a}+\frac{v^{2} t_{a}^{2}}{2 R_{2}}, \\
R_{I I}\left(t_{a}\right) & =R_{3}-\frac{\left[\left(x_{J}-x_{I}\right) v_{x}^{\prime}+\left(y_{J}-y_{I}\right) v_{y}^{\prime}\right]_{a}+\frac{\left(v_{x}^{\prime 2}+v_{y}^{\prime 2}\right)}{2 R_{3}} t_{a}^{2},}{R_{3}} \\
R_{I A_{1}}\left(t_{a}\right) & =R_{4}+\frac{\left[\left(v_{x}^{\prime}-v\right)\left(x_{I}+\left(v_{x}^{\prime} D / v\right)\right)+\left(y_{I}+\left(v_{y}^{\prime} D / v\right)\right) v_{y}^{\prime}\right]\left(t_{a}-(D / v)\right)}{R_{4}}+\frac{\left[\left(v-v_{x}^{\prime}\right)^{2}+v_{y}^{\prime 2}\right]}{2 R_{4}}\left(t_{a}-\frac{D}{v}\right)^{2}, \\
R_{I A_{2}}\left(t_{a}\right) & =R_{4}+\frac{\left[x_{I}\left(v_{x}^{\prime}-v\right)+y_{I} v_{y}^{\prime}\right]}{R_{4}} t_{a}+\frac{\left[\left(v-v_{x}^{\prime}\right)^{2}+v_{y}^{\prime 2}\right]}{2 R_{4}} t_{a}^{2}, \\
R_{I A_{3}}\left(t_{a}\right) & =R_{4}+\frac{\left[\left(v_{x}^{\prime}-v\right)\left(x_{I}-\left(v_{x}^{\prime} D / v\right)\right)+\left(y_{I}+\left(v_{y}^{\prime} D / v\right)\right) v_{y}^{\prime}\right]}{R_{4}}\left(t_{a}+\frac{D}{v}\right)+\frac{\left[\left(v-v_{x}^{\prime}\right)^{2}+v_{y}^{\prime 2}\right]}{2 R_{4}}\left(t_{a}+\frac{D}{v}\right)^{2}, \\
R_{4} & =\sqrt{x_{I}^{2}+y_{I}^{2}+H^{2}} . \\
R_{2} & =\sqrt{x_{J}^{2}+y_{J}^{2}+(H-h)^{2}}, \\
R_{3} & \sqrt{\left(x_{J}-x_{I}\right)^{2}+\left(y_{J}-y_{I}\right)^{2}+h^{2},}
\end{aligned}
$$

$$
R_{I A_{1}}\left(t_{a}\right)=\sqrt{\left(v t_{a}-D-x_{I}-v_{x}^{\prime} t_{a}\right)^{2}+\left(y_{I}+v_{y}^{\prime} t_{a}\right)^{2}+H^{2}} \text {, }
$$$$
R_{I A_{2}}\left(t_{a}\right)=\sqrt{\left(v t_{a}-x_{I}-v_{x}^{\prime} t_{a}\right)^{2}+\left(y_{I}+v_{y}^{\prime} t_{a}\right)^{2}+H^{2}},
$$$$
R_{I A_{3}}\left(t_{a}\right)=\sqrt{\left(v t_{a}+D-x_{I}-v_{x}^{\prime} t_{a}\right)^{2}+\left(y_{I}+v_{y}^{\prime} t_{a}\right)^{2}+H^{2}} .
$$

Equations (11)-(17) can be expanded as a Taylor series around $t_{a}$ at $0,0, D / v, 0$, and $-(D / v)$, and then they can be described as follows: 


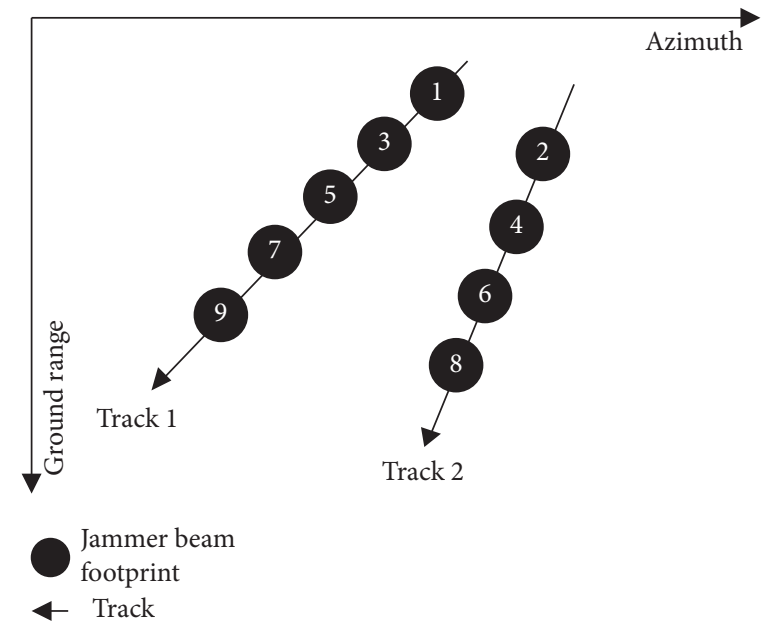

Figure 2: Tracks of the moving beam footprint.

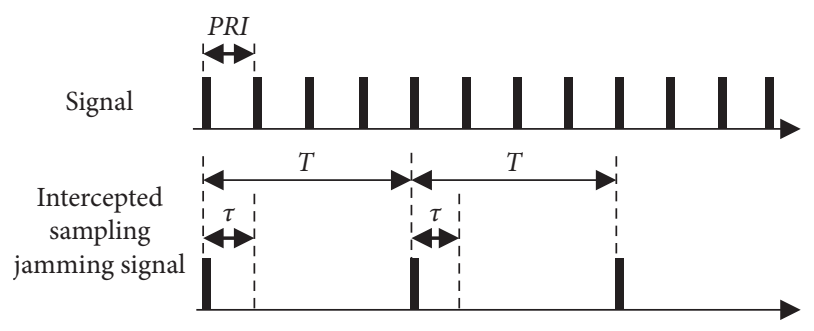

FIGURE 3: Jamming signal.

Considering $\quad v_{x} \ll v, \quad v_{y} \ll v, \quad D \ll R_{1}, \quad D \ll R_{3}$, and $D \ll R_{4}$. Then, the slant ranges for antennae $A_{1}$ and $A_{3}$ can be described as follows:

$$
\begin{aligned}
R_{F_{1}}\left(t_{a}\right)= & R_{A_{2} J}\left(t_{a}\right)+R_{J I}\left(t_{a}\right)+R_{I A_{2}}\left(t_{a}\right) \\
& +\frac{x_{I} D}{R_{4}}-\frac{v D}{R_{4}} t_{a}+\frac{D^{2}}{2 R_{4}}, \\
R_{F_{3}}\left(t_{a}\right)= & R_{A_{2} J}\left(t_{a}\right)+R_{J I}\left(t_{a}\right)+R_{I A_{2}}\left(t_{a}\right) \\
& -\frac{x_{I} D}{R_{4}}+\frac{v D}{R_{4}} t_{a}+\frac{D^{2}}{2 R_{4}} .
\end{aligned}
$$

The baseband jamming signal received by antennae $A_{1}$ and $A_{3}$ are, respectively, expressed as follows:

$$
\begin{aligned}
& S_{F_{1}}\left(t_{r}, t_{a}\right)=\sigma_{I} P\left(t_{a}\right) a_{r}\left(t_{r}-\frac{R_{F_{1}}\left(t_{a}\right)}{c}\right) a_{a z}\left(t_{a}-\frac{x_{F}^{\prime}}{v}\right) \exp \left[j \pi \gamma\left(t_{r}-\frac{R_{F_{1}}\left(t_{a}\right)}{c}\right)^{2}\right] \exp \left[-j \frac{2 \pi}{\lambda} R_{F_{1}}\left(t_{a}\right)\right], \\
& S_{F_{3}}\left(t_{r}, t_{a}\right)=\sigma_{I} P\left(t_{a}\right) a_{r}\left(t_{r}-\frac{R_{F_{3}}\left(t_{a}\right)}{c}\right) a_{a z}\left(t_{a}-\frac{x_{F}^{\prime}}{v}\right) \exp \left[j \pi \gamma\left(t_{r}-\frac{R_{F_{3}}\left(t_{a}\right)}{c}\right)^{2}\right] \exp \left[-j \frac{2 \pi}{\lambda} R_{F_{3}}\left(t_{a}\right)\right],
\end{aligned}
$$

where $x_{F}^{\prime}$ is the azimuth position of $F$ and $\sigma_{I}=G_{1} G_{2} \bar{\sigma}_{I}$, where $G_{1}$ is a jammer amplified factor and $\bar{\sigma}_{I}$ is an averaged RCS of the scattered point covered by $I$. Because amplitude of jamming signal is weakened by interrupted sampling modulation, $G_{2}=N$ is used to compensate amplitude. To generate $F$ as $P$, results of jamming signal and echo should be same after SAR, DPCA, and ATI technique.

\section{Analyze of Parameter Difference between Echo and Jamming Signal}

The proposed method depends on controlling factors, including the footprint's velocity and initial position, to form the false target as the moving target. By analyzing parameter difference between the false target and the moving target, such as position, Doppler rate expression, amplitude after 
DPCA technique, and initial azimuth position after ATI technique, factors are calculated for each track.
3.1. Range Position. Because $D \ll R_{1}$, where $R_{1}$ is $R_{A_{2} I}(0)$ and $R_{F_{1}}\left(t_{a}\right)$ approximately equals $R_{F_{3}}\left(t_{a}\right)$. Range position of $F$ will be expressed as follows [24]:

$$
R_{F}=\left.\frac{R_{F_{1}}\left(t_{a}\right)}{2}\right|_{t_{a}=0}=\frac{\sqrt{x_{J}^{2}+y_{J}^{2}+(H-h)^{2}}+\sqrt{\left(x_{J}-x_{I}\right)^{2}+\left(y_{J}-y_{I}\right)^{2}+h^{2}}+\sqrt{x_{I}^{2}+y_{I}^{2}+H^{2}}}{2} .
$$

Similarity, $P$ 's range position will be expressed as follows:

$$
R_{P}=\left.\frac{R_{P_{1}}\left(t_{a}\right)}{2}\right|_{t_{a}=0}=\sqrt{x_{P}^{2}+y_{P}^{2}+H^{2}}
$$

By adjusting $I$ 's initial range position, $\left|\left(R_{F}+c \Delta t_{j}\right) /\left(2-R_{P}\right)\right|<P_{r}$ should be satisfied, where $P_{r}$ is range resolution and $\Delta t_{j}$ is the delay of jammer. The jamming signal is not required to perform and store, and then the time delay within the jammer is too small to be noticed $[9,24,29]$.

3.2. Azimuth Position. Fs azimuth position is determined by the Doppler frequency at $t_{a}=0$ [24]. It will be expressed as follows:

$$
\begin{aligned}
x_{F}^{\prime} & =-\left.\frac{R_{F}}{2 v} \frac{d R_{F_{1}}\left(t_{a}\right)}{d t_{a}}\right|_{t_{a}=0} \\
& =\frac{R_{1}}{2 v}\left[\frac{\left[\left(x_{J}-x_{I}\right) v_{x}^{\prime}+\left(y_{J}-y_{I}\right) v_{y}^{\prime}\right]}{\sqrt{h^{2}+\left(x_{J}-x_{I}\right)^{2}+\left(y_{J}-y_{I}\right)^{2}}}+\frac{v x_{J}}{\sqrt{(H-h)^{2}+y_{J}^{2}+x_{J}^{2}}}-\frac{\left[v_{y}^{\prime} y_{I}-\left(v-v_{x}^{\prime}\right) x_{I}\right]}{\sqrt{x_{I}^{2}+y_{I}^{2}+H^{2}}}\right] .
\end{aligned}
$$

Similarity, $P$ 's azimuth position will be given as follows:

$$
x_{P}^{\prime}=-\left.\frac{R_{P}}{2 v} \frac{d R_{P_{1}}\left(t_{a}\right)}{d t_{a}}\right|_{t_{a}=0}=-\frac{\left[\left(v_{x}-v\right) x_{P}+y_{P} v_{y}\right]}{v} .
$$

By controlling I's velocity, $v_{x}^{\prime}$ and $v_{y}^{\prime},\left|x_{F}^{\prime}-x_{P}^{\prime}\right|<P_{a}$ should be satisfied, where $P_{a}$ is azimuth resolution.

3.3. Doppler Rate Mismatch. The second order term of slant ranges, which contains velocity of targets, will lead to defocusing in SAR images [24]. Then, F's Doppler rate expression will be given as follows:

$$
\begin{aligned}
K_{F}= & -\left.\frac{1}{\lambda} \frac{d^{2} R_{F_{1}}\left(t_{a}\right)}{d t_{a}^{2}}\right|_{t_{a}=0} \\
= & -\frac{1}{2 \lambda}\left[\frac{v^{2}}{\sqrt{(H-h)^{2}+y_{J}^{2}+x_{J}^{2}}}\right. \\
& \left.+\frac{v_{x}^{\prime 2}+v_{y}^{\prime 2}}{\sqrt{h^{2}+\left(x_{J}-x_{I}\right)^{2}+\left(y_{J}-y_{I}\right)^{2}}}+\frac{v_{y}^{\prime 2}+\left(v-v_{x}^{\prime}\right)^{2}}{\sqrt{x_{I}^{2}+y_{I}^{2}+H^{2}}}\right] .
\end{aligned}
$$

Similarity, $P$ 's Doppler rate expression will be given as follows:

$$
K_{P}=-\left.\frac{1}{\lambda} \frac{d^{2} R_{P_{1}}\left(t_{a}\right)}{d t_{a}^{2}}\right|_{t_{a}=0}=-\frac{v_{y}^{2}+\left(v-v_{x}\right)^{2}}{\lambda \sqrt{x_{P}^{2}+y_{P}^{2}+H^{2}}}
$$

By controlling I's velocity, $v_{x}^{\prime}$ and $v_{y}^{\prime}, K_{F} / K_{P} \leq 0.9$ should be satisfied.

3.4. DPCA Technique. After SAR processing, $F$ can be described as follows:

$$
\begin{aligned}
S_{1}\left(t_{r}, t_{a}+\frac{D}{v}\right)= & S_{F}\left(t_{r}, t_{a}\right) \exp \left[\frac{j 4 \pi D}{\lambda R_{1}}\left(x_{F}^{\prime}-\frac{x_{I}}{2}\right)\right], \\
S_{3}\left(t_{r}, t_{a}\right)= & S_{F}\left(t_{r}, t_{a}\right) \exp \left(\frac{j 4 \pi D}{\lambda R_{1}} \frac{x_{I}}{2}\right), \\
S_{F}\left(t_{r}, t_{a}\right)= & \sigma_{F}\left(1-\frac{\left|t_{a}-\left(x_{F}^{\prime} / v\right)\right|}{T_{a}}\right)\left(1-\frac{\left|t_{r}-\left(R_{F} / c\right)\right|}{T_{r}}\right) \sin c \\
& \cdot\left[\pi B_{r}\left(t_{r}-\frac{R_{F}}{c}\right)\left(1-\frac{\left|t_{r}-\left(R_{F} / c\right)\right|}{T_{r}}\right)\right] \\
& \times\left\{\sum _ { n = - \infty } ^ { + \infty } \operatorname { s i n } c \left\{\pi\left[\frac{n}{T}+r_{a}\left(t_{a}-\frac{x_{F}^{\prime}}{v}\right)\right]\right.\right. \\
& \left.\left.\cdot\left(T_{a}-\left|\frac{x_{F}^{\prime}}{v}\right|\right)\right\} \exp \left(\frac{j \pi n t_{a}}{T}\right) \cdot \frac{\tau}{T} \sin c\left(\frac{\pi n \tau}{T}\right)\right\},
\end{aligned}
$$

where $B_{a}$ is the Doppler bandwidth. 
Because the interrupted sampling jamming signal forms multiple targets, the main target, $n=0$, is considered to simulate the moving target.

DPCA technique can detect slow moving targets by substracting the coregistered SAR images, which belong to $A_{1}$ and $A_{3}$ [9]. Flowchart of DPCA technique is shown in Figure $4[30,31]$.

$F$ s DPCA result is described as follows:

$$
\begin{aligned}
\left|S_{F D P C A}^{\prime}\left(t_{r}, t_{a}\right)\right| & =\left|S_{3}\left(t_{r}, t_{a}\right)-S_{1}\left(t_{r}, t_{a}+\frac{D}{v}\right)\right| \\
& =2\left|S_{F}\left(t_{r}, t_{a}\right)\right| \cdot\left|\sin \left[\frac{2 \pi D\left(x_{F}^{\prime}-x_{I}\right)}{\lambda R_{4}}\right]\right| .
\end{aligned}
$$

Similarity, after SAR processing, $P$ is described as follows:

$$
\begin{aligned}
S_{1}\left(t_{r}, t_{a}+\frac{D}{v}\right)= & S_{P}\left(t_{r}, t_{a}\right) \exp \left[\frac{j 4 \pi D}{\lambda R_{1}}\left(x_{P}^{\prime}-\frac{x_{P}}{2}\right)\right], \\
S_{3}\left(t_{r}, t_{a}\right)= & S_{P}\left(t_{r}, t_{a}\right) \exp \left(\frac{j 4 \pi D}{\lambda R_{1}} \frac{x_{P}}{2}\right), \\
\left|S_{P}\left(t_{r}, t_{a}\right)\right|= & \sigma_{p}\left(1-\frac{\left|\left(t_{a}-x_{P}^{\prime}\right) / v\right|}{T_{a}}\right)\left(1-\frac{\left|\left(t_{r}-R_{P}\right) / c\right|}{T_{r}}\right) \\
& \times \sin c\left[\pi B_{r} \frac{\left(t_{r}-R_{P}\right)}{c}\left(1-\frac{\left|\left(t_{r}-R_{P}\right) / c\right|}{T_{r}}\right)\right] \\
& \times \sin c\left[\pi B_{a}\left(t_{a}-\frac{x_{P}^{\prime}}{v}\right)\left(1-\frac{\left|\left(t_{a}-x_{P}^{\prime}\right) / c\right|}{T_{a}}\right)\right] .
\end{aligned}
$$

Then, $P$ 's DPCA result is expressed as follows:

$$
\left|S_{P}^{\prime}\left(t_{r}, t_{a}\right)\right|=2\left|S_{P}\left(t_{r}, t_{a}\right)\right| \cdot\left|\sin \left[\frac{2 \pi D\left(x_{P}^{\prime}-x_{P}\right)}{\lambda R_{1}}\right]\right| .
$$

Because the jammer is near the protected area, $R_{1} \approx R_{4}$ can be satisfied. Comparing (28) with (30), if the initial azimuth position of the footprint $x_{I}$ approximately equals that of moving target $x_{P}, F$ and $P$ have an approximate amplitude after DPCA technique. $\left|x_{I}-x_{P}\right|<P_{a}$ should be satisfied.

3.5. ATI Technique. ATI technique abstracts the interferometric phase information between two coregistered SAR images to resolve the relocated azimuth position of slow-moving targets [9]. Flowchart of ATI technique is shown in Figure 5 [30, 32].

$D, \lambda$, and $R_{1}$ and the phase of the false target are known parameters for SAR GMTI, and $x_{F}^{\prime}$ can be obtained from the SAR image. So, $F$ 's relocated azimuth position will be detected after ATI technique. $F$ is described as follows:

$$
\begin{aligned}
S_{\text {FATI }}^{\prime}\left(t_{r}, t_{a}\right) & =S_{3}\left(t_{r}, t_{a}\right) \cdot S_{1}^{*}\left(t_{r}, t_{a}+\frac{D}{v}\right) \\
& =\left|S_{F}\left(t_{r}, t_{a}\right)\right|^{2} \cdot \exp \left[\frac{j 4 \pi D\left(x_{F}^{\prime}-x_{I}\right)}{\lambda R_{1}}\right],
\end{aligned}
$$

where $*$ represents the complex conjugate.

$F$ s interferometric phase is given by

$$
\widehat{\phi}_{F}=\arg \left\{S_{F A T I}^{\prime}\left(t_{r}, t_{a}\right)\right\}=\frac{4 \pi D\left(x_{F}^{\prime}-x_{I}\right)}{\lambda R_{1}},
$$

where $\arg \{\cdot\}$ represents the acquiring phase term operation.

$F$ s estimate azimuth position will be relocated to

$$
\widehat{x}_{F}=x_{F}^{\prime}-\frac{\lambda R_{1}}{4 \pi D} \widehat{\phi}_{F} \approx x_{I} .
$$

Similarly, $P$ is described as follows:

$$
\begin{aligned}
S_{P A T I}^{\prime}\left(t_{r}, t_{a}\right) & =S_{3}\left(t_{r}, t_{a}\right) \cdot S_{1}^{*}\left(t_{r}, t_{a}+\frac{D}{v}\right) \\
& =\left|S_{F}\left(t_{r}, t_{a}\right)\right|^{2} \cdot \exp \left[\frac{j 4 \pi D\left(x_{P}^{\prime}-x_{P}\right)}{\lambda R_{1}}\right] .
\end{aligned}
$$

$P$ 's interferometric phase is given by

$$
\widehat{\phi}_{P}=\arg \left\{S_{\text {FATI }}^{\prime}\left(t_{r}, t_{a}\right)\right\}=\frac{4 \pi D\left(x_{P}^{\prime}-x_{P}\right)}{\lambda R_{1}} .
$$

$P$ 's the relocated azimuth position will be estimated by

$$
\widehat{x}_{P}=x_{P}^{\prime}-\frac{\lambda R_{1}}{4 \pi D} \widehat{\phi}_{P} \approx x_{P} .
$$

Based on the above analysis, if initial azimuth position of the footprint $x_{I}$ approximately equals that of moving target $x_{P}, F$ and $P$ have an approximate relocated azimuth position after ATI technique.

3.6. Realization. The steps of generating a verisimilar false moving target will be represented as follows. First, I's initial azimuth position, $x_{I}$, should be adjusted to be equal to that of $P$. Second, by controlling $I$ 's initial position in the ground range direction, $y_{I}, F$ s range position is similar to that of $P$ in images. Third, by controlling $I$ 's velocity, $v_{x}^{\prime}$ and $v_{y}^{\prime}, F$ s Doppler rate mismatch and the azimuth position will be similar to those of $P, K_{F} \approx K_{P}$ and $x_{F}^{\prime} \approx x_{P}^{\prime}$. Henceforth, according to the calculated initial position and velocity of jamming beam footprint, the track will be decided. Fifth, according to the azimuth velocity and number of false targets, which intend to generate, the time interval $T$ between the adjacent jamming footprints is calculated. The beam interrupted sampling illuminates the ground along the track. Then, $F$ has the same amplitude and the relocated azimuth position as $P$. 


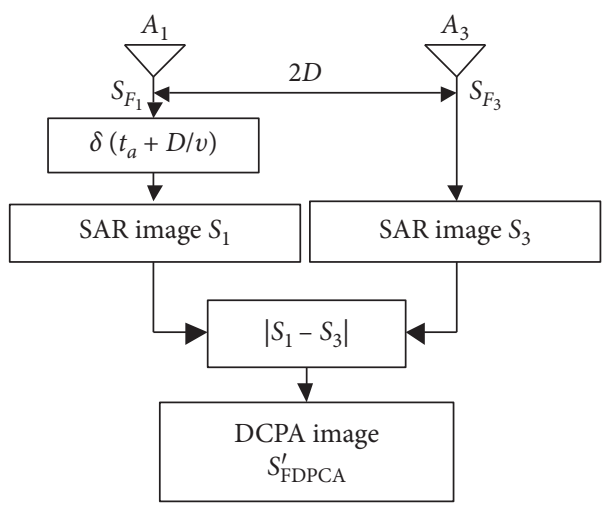

Figure 4: Flowchart of DPCA technique.

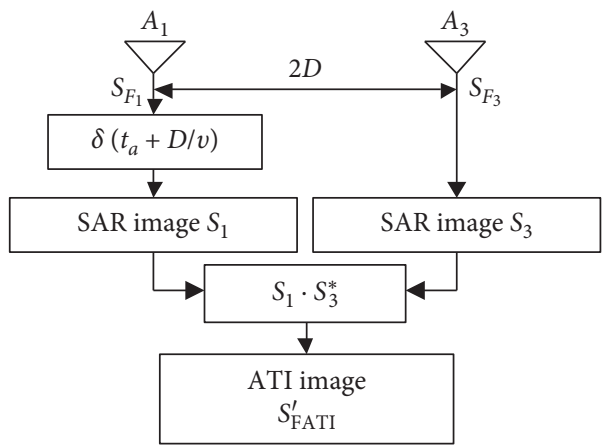

FIgURE 5: Flowchart of ATI technique.

\section{Simulation}

Parameters of radar are listed in Table 1.

4.1. A False Moving Target Simulation. In this section, we examine that a false target generated with the proposed method can be imaged as a moving target, and detected results of the simulated moving target are provided for contrast analysis. The moving target's initial position is $(10 \mathrm{~m}, 10,050 \mathrm{~m}, 0 \mathrm{~m})$, and its velocity are $v_{x}=0.5 \mathrm{~m} / \mathrm{s}$ and $v_{y}=0.5 \mathrm{~m} / \mathrm{s}$. A jammer is the assumed set at $(0 \mathrm{~m}, 10,000 \mathrm{~m}$, $10 \mathrm{~m})$. The jammer beam footprint's initial position is $(10 \mathrm{~m}$, $10,044.3 \mathrm{~m}, 0 \mathrm{~m})$ and its velocity are $v_{x}^{\prime}=1.1 \mathrm{~m} / \mathrm{s}$ and $v_{y}^{\prime}=0.2 \mathrm{~m} / \mathrm{s}$.

The SAR imaging results of the real target and the false target are shown in Figure 6, and their azimuth sectional plots are shown in Figure 7. They are well focused and imaged in the SAR image, and they are almost the same, which verifies the effectiveness of the proposed method. In addition, the errors, which contain position error, the impulse response width (IRW) error and the peak side lobe ratio (PSLR) error, are listed in Table 2 [29]. The positions of the real target and false target are the same. The error of IRW is too small to be recognized by the radar. PSLR of false target is slightly better than that of the moving target, which contributes to mislead radar decision. So, the proposed method has the ability to generate a verisimilar false target against SAR.
TABle 1: Parameters of radar.

\begin{tabular}{lcc}
\hline Parameters & Symbol & Value \\
\hline SAR velocity & $v$ & $200 \mathrm{~m} / \mathrm{s}$ \\
Pulse repetition frequency & PRF & $1,024 \mathrm{~Hz}$ \\
Effective baseline & $D$ & $0.4 \mathrm{~m}$ \\
Altitude & $H$ & $10,000 \mathrm{~m}$ \\
Pulse duration & $T_{r}$ & $10 \mu \mathrm{s}$ \\
Signal bandwidth & $B_{r}$ & $200 \mathrm{MHz}$ \\
Target exposure time & $T_{a}$ & $1.8 \mathrm{~s}$ \\
Frequency & $f_{0}$ & $9.6 \mathrm{GHz}$ \\
\hline
\end{tabular}

The DPCA results are shown in Figure 8, and their azimuth sectional plots are shown in Figure 9. Targets are well focused and imaged in the DPCA image, which verifies the effectiveness of the proposed method. The quality results are listed in Table 3.

Imaging quality parameters of the moving target is similar to those of the false target. So, the proposed method has ability to generate a false target against DPCA technique.

Next, Figure 10 shows interferograms of targets utilizing ATI technique. Then, the relocated azimuth position can be estimated.

After ATI technique, their relocated azimuth positions are detected and are listed in Table 4. The relocated azimuth position error between the false target and the moving target is too small to be noticed. So, the proposed method has the ability to generate a false target which is similar to a moving target against ATI technique. 


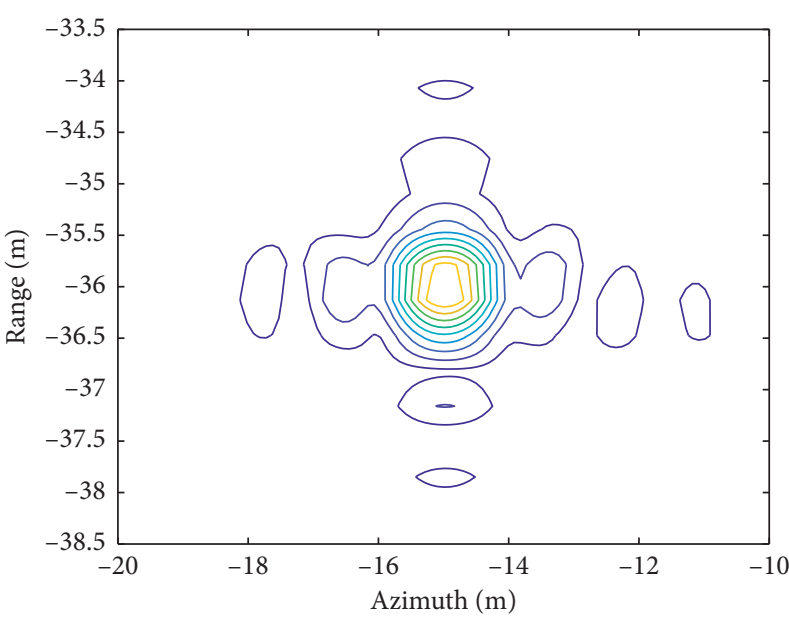

(a)

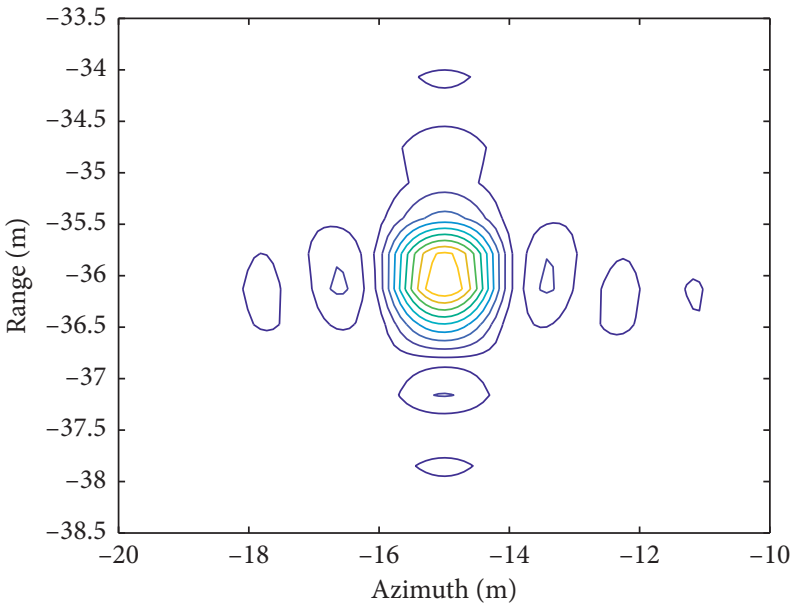

(b)

Figure 6: SAR images of the real target and the false target. (a) Contour plots of the real target. (b) Contour plots of the false target.

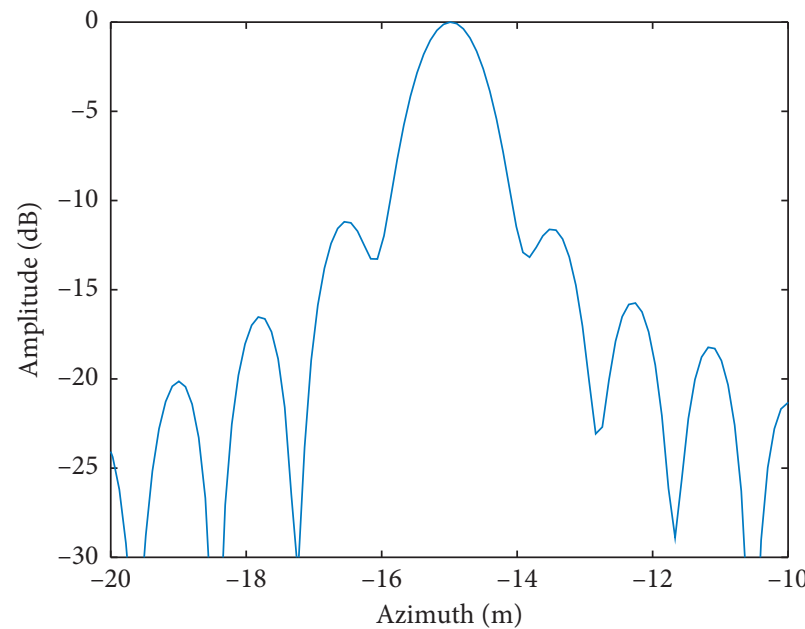

(a)

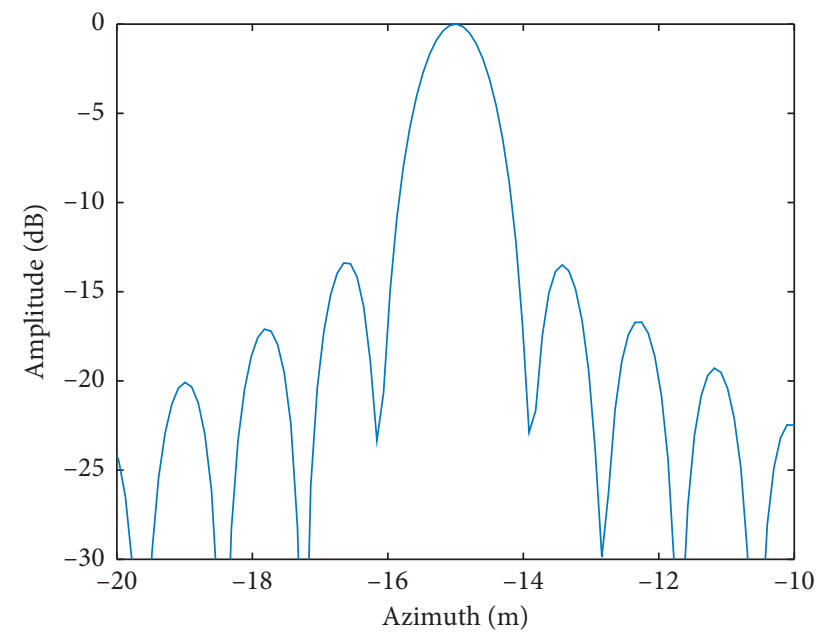

(b)

FIgURE 7: Azimuth sectional plots of SAR images: (a) the real target and (b) the false target.

TABLE 2: Imaging quality parameters of SAR images.

\begin{tabular}{lccc}
\hline Parameters & Moving target & False target & \\
\hline Range position & $36.13 \mathrm{~m}$ & $36.13 \mathrm{~m}$ & Error \\
Azimuth position & $-14.99 \mathrm{~m}$ & $-14.99 \mathrm{~m}$ & $0 \mathrm{~m}$ \\
IRW & $2.24 \mathrm{~m}$ & $2.25 \mathrm{~m}$ & $0 \mathrm{~m}$ \\
PSLR & $11.19 \mathrm{~dB}$ & $13.43 \mathrm{~dB}$ & $0.01 \mathrm{~m}$ \\
\hline
\end{tabular}

4.2. Multiple False Moving Target Simulation. In this part, to further represent jamming effect, comparative experiments are achieved by utilizing the method in $[9,21]$, which is a traditional deception jamming based on signal jammer and is able to generate multiple false targets. Two moving targets are simulated by utilizing the traditional method and the proposed method to generate two false moving targets. Their initial azimuth positions are $30 \mathrm{~m}$ and $40 \mathrm{~m}$. The DPCA results are shown in Figure 11.
Figure 12 shows interferograms of false targets detected by ATI technique. Then, the velocity in ground direction and the relocated azimuth position will be detected.

Although DPCA results are the same in Figure 11, the interferograms are different in Figure 12. The false targets, which are generated with the traditional deception jamming method, all have the similar relocated azimuth position, as shown in Table 5, which is difficult to simulate moving targets and misleads the decision-making of the radar. However, false targets generated with the proposed method 


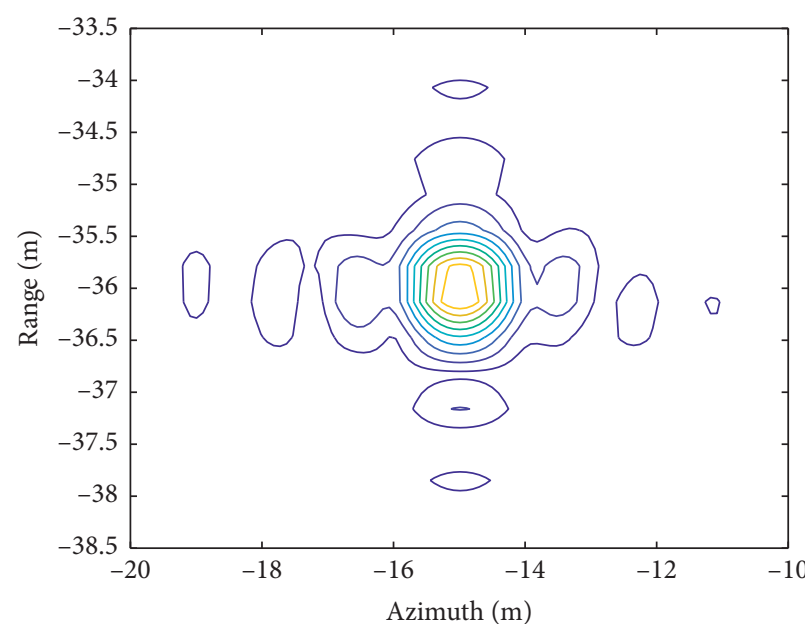

(a)

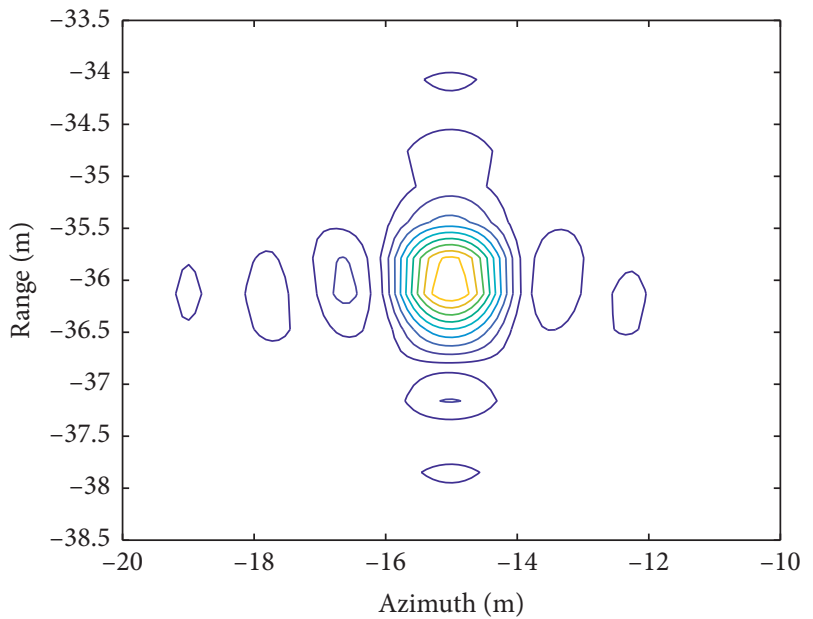

(b)

FIGURE 8: DPCA images of the real target and the false target. (a) Contour plots of the real target. (b) Contour plots of the false target.

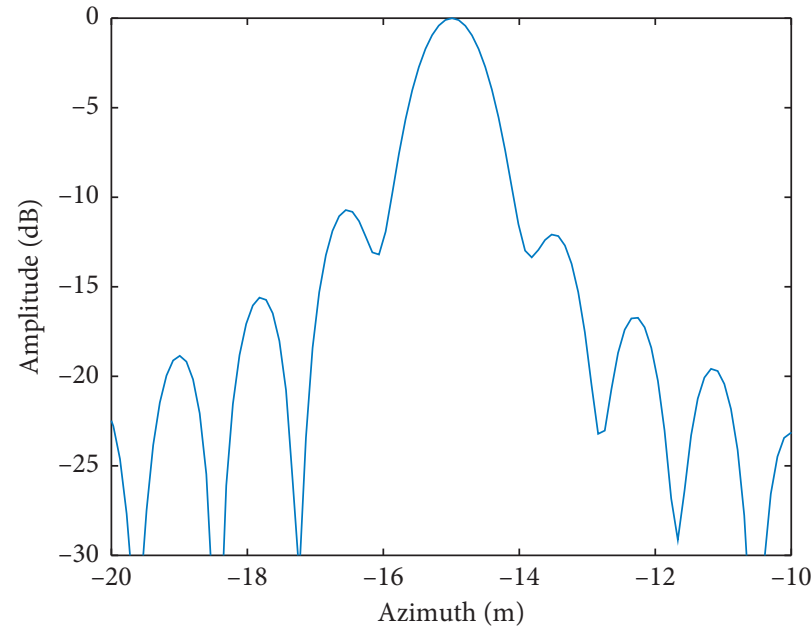

(a)

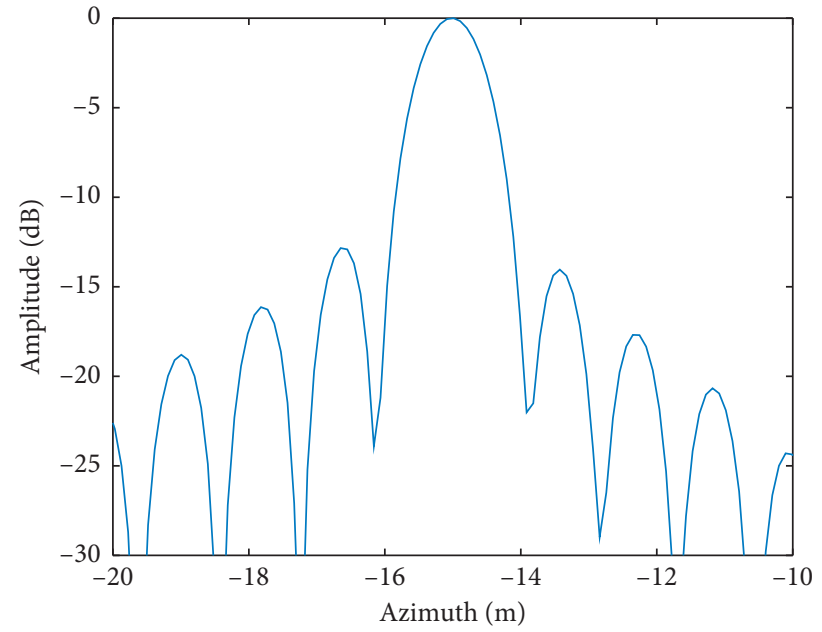

(b)

FIgURE 9: Azimuth sectional plots of DPCA images: (a) the real target and (b) the false target.

TABLE 3: Imaging quality parameters of DPCA images.

\begin{tabular}{lccc}
\hline Parameters & Moving target & False target & \\
\hline Range & $36.13 \mathrm{~m}$ & $36.13 \mathrm{~m}$ & Error \\
Azimuth & $-14.99 \mathrm{~m}$ & $-14.99 \mathrm{~m}$ & $0 \mathrm{~m}$ \\
IRW & $2.24 \mathrm{~m}$ & $2.25 \mathrm{~m}$ & $0 \mathrm{~m}$ \\
PSLR & $11.19 \mathrm{~dB}$ & $12.84 \mathrm{~dB}$ & $0.01 \mathrm{~m}$ \\
\hline
\end{tabular}




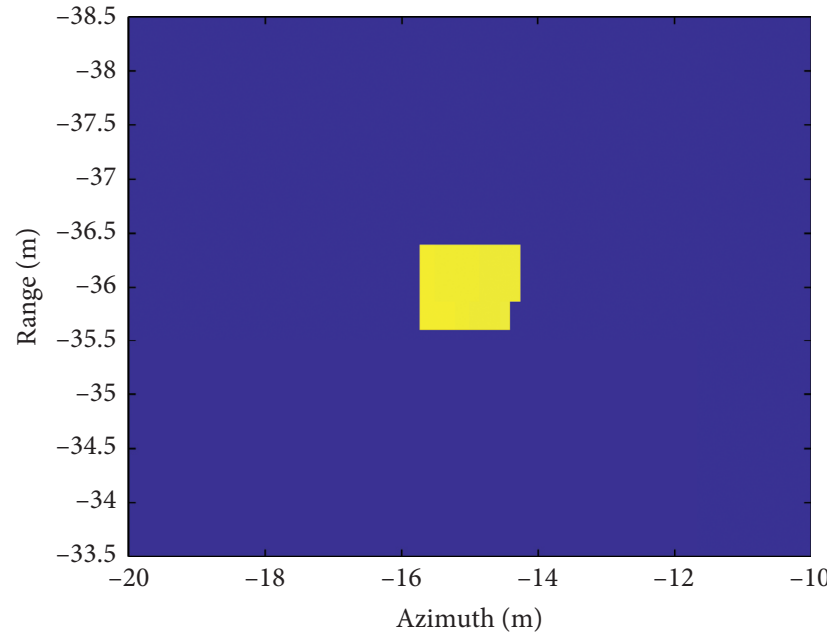

(a)

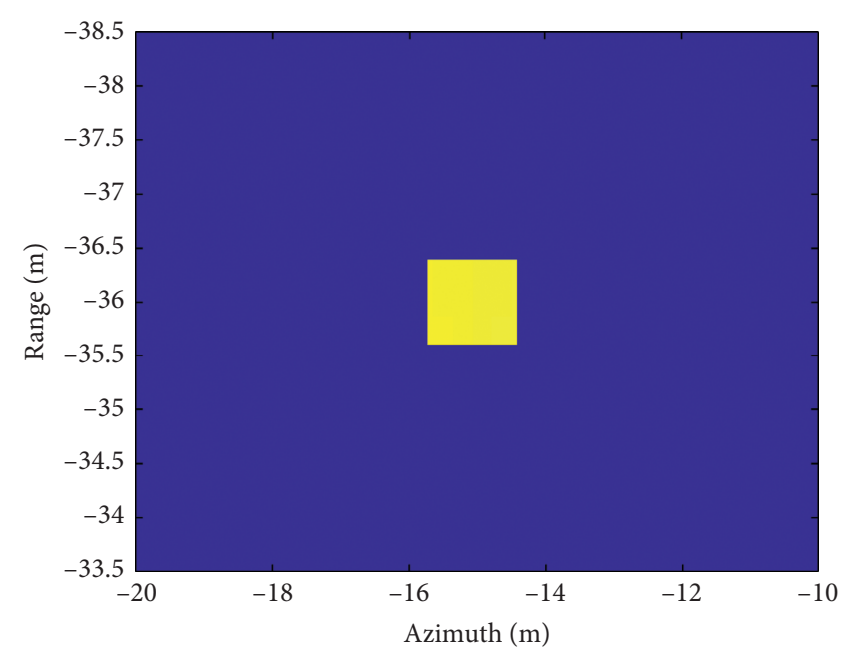

(b)

FIGURE 10: Interferograms of the real target and the false target. (a) Interferogram of the real target. (b) Interferogram of the false target.

TABLE 4: Detected result of the false target.

\begin{tabular}{lr}
\hline Index & The relocated azimuth position (m) \\
\hline Moving target & 10.16 \\
False moving target & 10.17 \\
Error & 0.01 \\
\hline
\end{tabular}

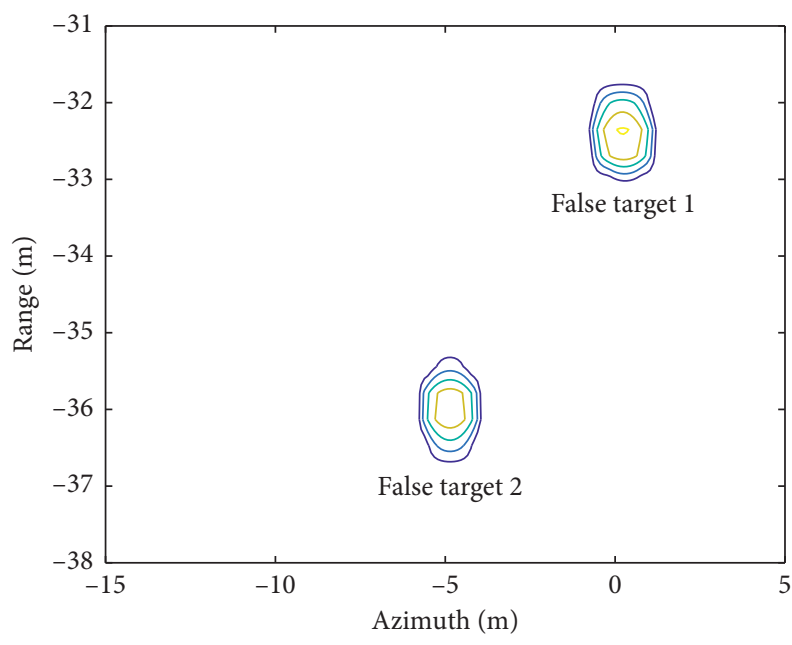

(a)

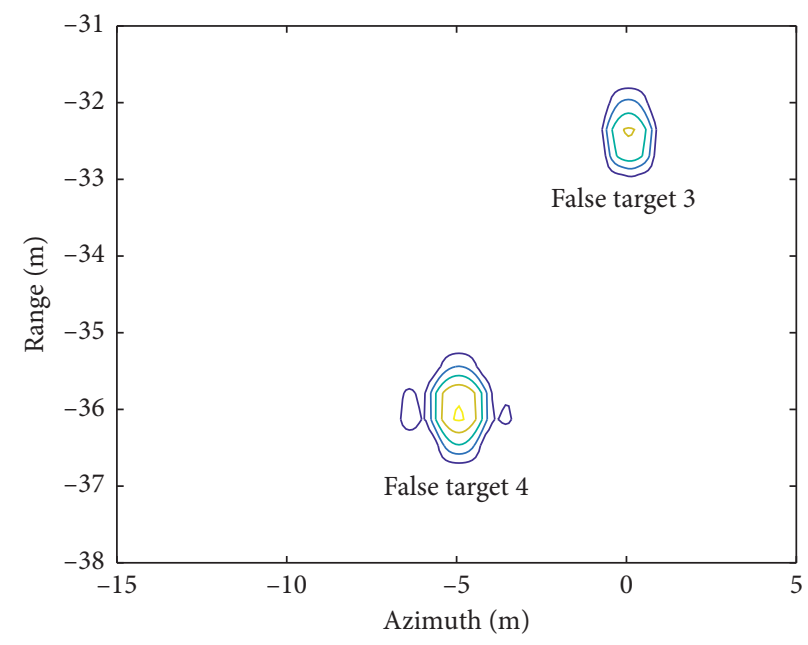

(b)

FIGURE 11: DPCA images of false moving targets. (a) Contour plots of false moving targets generated by the traditional method. (b) Contour plots of false moving targets generated by the proposed method. 


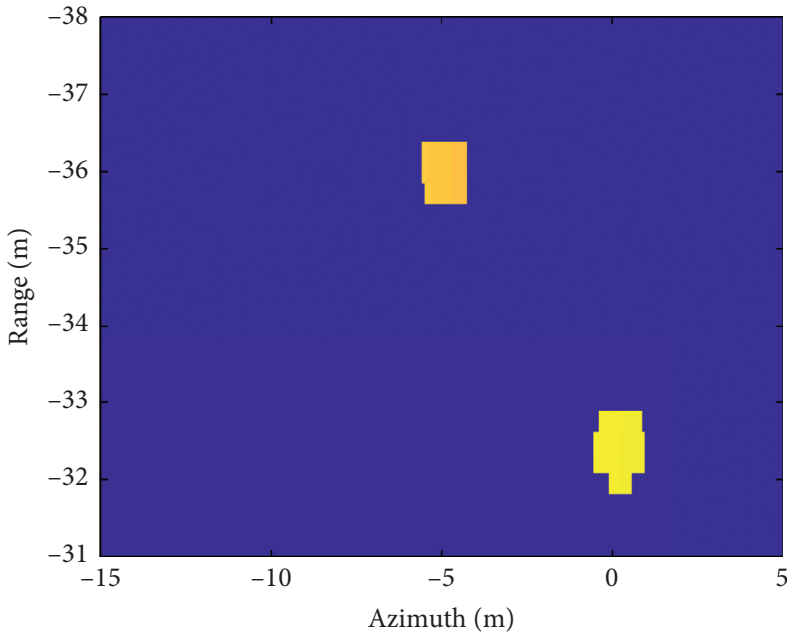

(a)

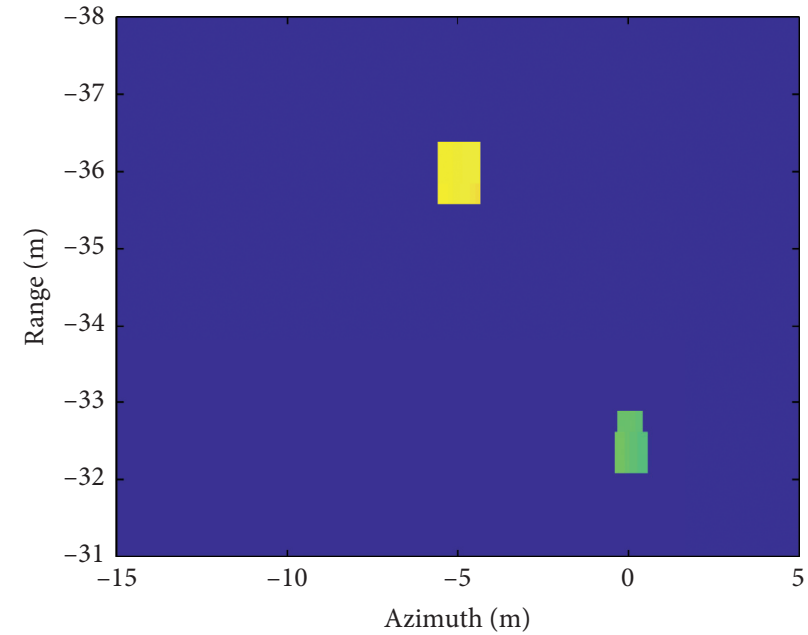

(b)

FIGURE 12: Interferogram of false moving targets. (a) Interferogram of false moving targets generated by the traditional method. (b) Interferogram of false moving targets generated by the proposed method.

TABLE 5: Detected result of false targets.

\begin{tabular}{lc}
\hline Target number & The relocated azimuth position $(\mathrm{m})$ \\
\hline False target 1 & 9.85 \\
False target 2 & 10.20 \\
False target 3 & 30.25 \\
False target 4 & 40.26 \\
\hline
\end{tabular}

have the different relocated azimuth positions, which are similar to the initial azimuth positions of the moving targets. So, the proposed method is able to generate multiple false moving targets.

\section{Conclusions}

An important problem is how to generate false targets with the same initial azimuth positions as those of moving targets. To solve this problem, a scattered wave deception jamming method with a controllable jammer antenna is proposed. The improvement is that a stationary jammer is set affront of the protected area with a controllable antenna to generate very similar moving targets by controlling jammer beam footprint's velocity and initial position. The footprint moves along the different calculated tracks. For each track, slant ranges of the jamming signal are changed varying with different PRI, and the movement of the jammer antenna beam would introduce a Doppler component. By analyzing parameter difference between the false target and the moving target, the velocity and initial position of the footprint are calculated, and then the track will be determined. In addition, during the time interval between adjacent intercepted slice, the jammer beam can be controlled to illuminated different tracks, and then targets are generated. Simulation experiments use the jamming quality parameters, which contain position information, IRW, PSLR, and the relocated azimuth position, to evaluate jamming effect. The effectiveness of the method is verified by experiment results.

\section{Data Availability}

No data were used to support this study because all experiments were simulated based on equations of the proposed method.

\section{Conflicts of Interest}

The authors declare that they have no conflicts of interest.

\section{References}

[1] Z. Chen, Y. Zhou, L. Zhang, C. Lin, Y. Huang, and S. Tang, "Ground moving target imaging and analysis for near-space hypersonic vehicle-borne synthetic aperture radar system with squint angle," Remote Sensing, vol. 10, no. 12, p. 1966, 2018.

[2] Y. Zhao, S. Han, J. Yang, L. Zhang, H. Xu, and J. Wang, “A novel approach of slope detection combined with lv's distribution for airborne SAR imagery of fast moving targets," Remote Sensing, vol. 10, no. 5, p. 764, 2018.

[3] X. Shi, F. Zhou, S. Yang, Z. Zhang, and T. Su, "Automatic target recognition for synthetic aperture radar images based on super-resolution generative adversarial network and deep convolutional neural network," Remote Sensing, vol. 11, no. 2, p. 135, 2019.

[4] W. Fan, F. Zhou, M. Tao et al., "Interference mitigation for synthetic aperture radar based on deep residual network," Remote Sensing, vol. 11, no. 14, p. 1654, 2019.

[5] S. Tanelli, S. L. Durden, and M. P. Johnson, "Airborne demonstration of DPCA for velocity measurements of distributed targets," IEEE Geoscience and Remote Sensing Letters, vol. 13, no. 10, pp. 1415-1419, 2016.

[6] E. Chapin and C. W. Chen, "Along-track interferometry for ground moving target indication," IEEE Aerospace and Electronic Systems Magazine, vol. 23, no. 6, pp. 19-24, 2008.

[7] B. Liu, K. Yin, Y. Li, F. Shen, and Z. Bao, "An improvement in multichannel SAR-GMTI detection in heterogeneous environments," IEEE Transactions on Geoscience and Remote Sensing, vol. 53, no. 2, pp. 810-827, 2015. 
[8] B. Filippo, "COSMO-SkyMed staring spotlight SAR data for micro-motion and inclination angle estimation of ships by pixel tracking and convex optimization," Remote Sensing, vol. 11, no. 7, p. 766, 2019.

[9] Q. Sun, T. Shu, K.-B. Yu, and W. Yu, "A novel deceptive jamming method against two-channel SAR-GMTI based on two jammers," IEEE Sensors Journal, vol. 19, no. 14, pp. 5600-5610, 2019.

[10] L. Huang, C. Dong, Z. Shen, and G. Zhao, "The influence of rebound jamming on SAR GMTI," IEEE Geoscience and Remote Sensing Letters, vol. 12, no. 2, pp. 399-403, 2015.

[11] H. Shi, Y. Zhou, and J. Chen, "An method of SAR GMTI in the presence of blanketing jamming," in Proceedings of the 1st Asian Pacific Conference Synthetic Aperture Radar (APSAR 2007), Huangshan, China, November 2007.

[12] J. Xu, G. Liao, S. Zhu, and H. C. So, "Deceptive jamming suppression with frequency diverse MIMO radar," Signal Processing, vol. 113, pp. 9-17, 2015.

[13] J. Xu, J. Kang, G. Liao, and H. So, "Mainlobe deceptive jammer suppression with FDA-MIMO radar," in Proceedings of the IEEE Sensor Array \& Multichannel Signal Processing Workshop, Sheffield, UK, July 2018.

[14] J. Zhang, D. Dai, S. Xing, S. Xiao, and B. Pang, "A novel barrage repeater jamming against SAR-GMTI," in Proceedings of the 2016 10th European Conference on Antennas and Propagation (EuCAP), Davos, Switzerland, April 2016.

[15] F. Zhou, G. Sun, X. Bai, and Z. Bao, "A novel method for adaptive SAR barrage jamming suppression," IEEE Geoscience and Remote Sensing Letters, vol. 9, no. 2, pp. 292-296, 2012.

[16] B. Zhao, L. Huang, J. Li, M. Liu, and J. Wang, "Deceptive SAR jamming based on 1-bit sampling and time-varying thresholds," IEEE Journal of Selected Topics in Applied Earth Observations and Remote Sensing, vol. 11, no. 3, pp. 939-950, 2018.

[17] X. Chang and C. Dong, "A barrage noise jamming method based on double jammers against three channel SAR GMTI," IEEE Access, vol. 7, pp. 18755-18763, 2019.

[18] Q. Sun, T. Shu, K.-B. Yu, and W. Yu, "Efficient deceptive jamming method of static and moving targets against SAR," IEEE Sensors Journal, vol. 18, no. 9, pp. 3610-3618, 2018.

[19] X. Shi, F. Zhou, B. Zhao, M. Tao, and Z. Zhang, "Deception jamming method based on micro-Doppler effect for vehicle target," IET Radar Sonar and Navigation, vol. 10, no. 6, pp. 1071-1079, 2016.

[20] X. Li, B. Deng, Y. Qin, H. Wang, and Y. Li, "The influence of target micromotion on SAR and GMTI," IEEE Transactions on Geoscience and Remote Sensing, vol. 49, no. 7, pp. 27382751, 2011.

[21] X. F. Wu, X. F. Wang, and J. X. Ling, "Modulation jamming method for high-vivid false uniformly-moving targets against SAR-GMTI," Journal of Astronautics, vol. 33, no. 10, pp. 1472-1479, 2012.

[22] J. Zhang, Study on distributed cooperative jamming techniques against multichannel SAR, Ph.D. dissertation, National University of DefenseTechnology, Changsha, Hunan, China, 2016.

[23] J. Zhang, Y. Li, D. Dai, S. Xing, and S. Xiao, "Three-dimensional deceptive scene generation against single-pass InSAR based on coherent transponders," IET Radar, Sonar \& Navigation, vol. 10, no. 3, pp. 477-487, 2016.

[24] B. Zhao, Z. Bao, F. Zhou, M. Tao, and Z. Zhang, "Improved method for synthetic aperture radar scattered wave deception jamming," IET Radar, Sonar \& Navigation, vol. 8, no. 8, pp. 971-976, 2014.
[25] M. Fang, D. Bi, and A. Shen, "Countering performace analysis of scatter-wave jamming against multi-Channel SAR-GMTI," in Proceedings of the 2016 CIE International Conference on Radar (RADAR), Guangzhou, China, October 2016.

[26] L. Jia, X. Jia, Y. He, and C. Huang, "Analysis on the effects of rebound jamming on InSAR imaging," Electronic Information Warfare Technology, vol. 27, pp. 42-48, 2012.

[27] C. Dong and X. Chang, "A novel scattered wave deception jamming against three channel SAR GMTI," IEEE Access, vol. 6, pp. 53882-53889, 2018.

[28] C. Zhou, Q. Liu, and X. Chen, "Parameter estimation and suppression for DRFM-based interrupted sampling repeater jammer," IET Radar, Sonar \& Navigation, vol. 12, no. 1, pp. 56-63, 2018.

[29] B. Zhao, F. Zhou, and Z. Bao, "Deception jamming for squint SAR based on multiple receivers," IEEE Journal of Selected Topics in Applied Earth Observations and Remote Sensing, vol. 8, no. 8, pp. 3988-3998, 2015.

[30] C. Shen and C. Livingstone, "A comparison of displaced phase centre antenna and along-track interferometry techniques for RADARSAT-2 ground moving target indication," Canadian Journal of Remote Sensing, vol. 31, no. 1, pp. 37-51, 2005.

[31] D. Cerutti-Maori and I. Sikaneta, "A generalization of DPCA processing for multichannel SAR/GMTI radars," IEEE Transactions on Geoscience and Remote Sensing, vol. 51, no. 1, pp. 560-572, 2013.

[32] C. H. Gierull, I. Sikaneta, and D. Cerutti-Maori, “Two-step detector for RADARSAT-2's experimental GMTI mode," IEEE Transactions on Geoscience and Remote Sensing, vol. 51, no. 1, pp. 436-454, 2013. 